\title{
A checklist of the helminth parasites of invasive murid rodents in Mexico
}

\author{
Jesús Alonso Panti-May ${ }^{1}$, Alejandra Duarte-Jiménez ${ }^{1}$, Silvia F. Hernández-Betancourt ${ }^{1}$, and Roger Iván Rodríguez-Vivas ${ }^{1 *}$ \\ 'Facultad de Medicina Veterinaria y Zootecnia, Campus de Ciencias Biológicas y Agropecuarias, Universidad Autónoma de Yu- \\ catán, Mérida, Yucatán, México. Email: panti.alonso@gmail.com (JAP-M), alejandraduarte1996@gmail.com (AD-J), hbetanc@ \\ correo.uady.mx (SFH-B), rvivas@correo.uady.mx (RIR-V) \\ *Corresponding author
}

\begin{abstract}
El presente trabajo provee un listado helmintológico actualizado de los roedores múridos invasores (Mus musculus, Rattus norvegicus, $R$. rattus y Rattus sp.) en México, que incluye 35 taxa de helmintos (3 tremátodos, 7 céstodos, 2 acantocéfalos y 23 nematodos). Los registros helmintológicos hasta mayo de 2020, provienen de 18 estudios procedentes de siete estados del país. Se incluye información de los registros acerca de los hábitats, estadios de desarrollo, localidades de registro, hospedadores, colecciones helmintológicas donde se encuentran depositados los especímenes, prevalencias y referencias bibliográficas, cuando estuvieron disponibles. Finalmente, se adiciona un nuevo registro de localidad para el nematodo Hassalstrongylus musculi en Yucatán.

The present work provides an updated checklist of helminth species infecting invasive murid rodents (Mus musculus, Rattus norvegicus, $R$. rattus, and Rattus sp.) in Mexico, including 35 helminth taxa ( 3 trematodes, 7 cestodes, 2 acanthocephalans, and 23 nematodes). The helminthological records were compiled from 18 studies conducted in seven Mexican states up to May 2020. Information on habitats, life stages, geographical locations, hosts, helminthological collections, prevalences, and bibliographic references are included, when available. Finally, a new locality record of the nematode Hassalstrongylus musculi in Yucatan is provided.
\end{abstract}

Keywords: Helminthological record; Mus musculus; Rattus norvegicus; Rattus rattus.

(c) 2021 Asociación Mexicana de Mastozoología, www.mastozoologiamexicana.org

\section{Introducción}

Los roedores representan aproximadamente el $39 \%$ de los mamíferos del mundo (Burgin et al. 2018). A pesar de este porcentaje, pocas especies son consideradas como invasoras en hábitats naturales, urbanos y rurales (Stenseth et al. 2003). El ratón doméstico Mus musculus, la rata café o noruega Rattus norvegicus y la rata negra o de los tejados Rattus rattus (Rodentia: Muridae), son consideradas las especies invasoras más importantes a nivel mundial. Estos roedores han causado extinciones de plantas, invertebrados y pequeños mamíferos en numerosas islas del mundo (Leitschuh et al. 2018), y pérdidas económicas a nivel mundial por las toneladas de cereales que consumen o degradan anualmente (Singleton et al. 2010), así como mortalidades y morbilidades en los seres humanos causadas por patógenos zoonóticos como los helmintos (e. g., Angiostrongylus cantonensis, Hymenolepis diminuta) que éstos hospedan (Meerburg et al. 2009).

En México, los estudios helmintológicos en pequeños roedores iniciaron en la década de 1930 (Ochoterena y Caballero y Caballero 1932; Chitwood 1938; Caballero y Caballero 1939). En roedores invasores los primeros registros helmintológicos fueron publicados por Caballero y Caballero (1939) quien registró tres especies de céstodos (Hydatigera taeniaeformis, H. diminuta y Rodentolepis nana) y un nematodo (Heterakis spumosa) en $R$. norvegicus en la Ciudad de México. Recientemente, García-Prieto et al. (2012) compilaron el listado de helmintos de los mamíferos silvestres de México, incluyendo aquellos reportados en roedores invasores. En este estudio se listaron 10 especies de helmintos en $M$. musculus y $R$. rattus y 12 especies en $R$. norvegicus. Sin embargo, este trabajo carece de los registros más recientes y posteriores al año 2012, especialmente aquellos realizados en el sureste de México, donde los estudios en roedores múridos invasores se han incrementado debido a su papel como reservorios de helmintos zoonóticos (e. g.,Panti-May et al. 2015, 2020a).

El objetivo del presente trabajo es actualizar el listado de helmintos parásitos de roedores múridos invasores en México, compilando los trabajos más recientes y añadiendo un nuevo registro no publicado para el estado de Yucatán.

\section{Materiales y métodos}

Se realizó una búsqueda electrónica de literatura sobre helmintos parásitos de roedores múridos invasores en México. Para ello se consultaron tres bases de datos electrónicas (Google Scholar, PubMed y Web of Science) utilizando las palabras clave "helminto", "céstodo", "nematodo", "tremátodo", "acantocéfalo", "parásito", "Mus musculus", "Rattus rattus", "Rattus norvegicus", "roedor invasor", "roedor comensal", "roedor sinantrópico" y "México" en español e inglés. La búsqueda incluyó trabajos desde el año 2012 hasta mayo de 2020. La revisión incluyó estudios con infecciones naturales en poblaciones sinantrópicas o comensales de roedores invasores; no se incluyen infecciones experimentales o naturales en animales de líneas o cepas usadas en laboratorios o centros comerciales de 
mascotas. Los registros de los trabajos identificados en la búsqueda electrónica fueron añadidos al listado helmintológico publicado por García-Prieto et al. (2012). Las referencias bibliográficas de este último trabajo fueron consultadas para confirmar la información en la fuente original.

Los registros helmintológicos fueron agrupados por filo, y ordenados alfabéticamente a nivel familia, género y especie. Para cada especie de helminto se presenta información sobre el hábitat, estado de desarrollo, localidades de registro, hospedadores, colecciones helmintológicas donde están depositados los ejemplares, prevalencias y referencias bibliográficas, siempre y cuando estén disponibles. EI registro referenciado en el presente estudio proviene de un muestreo realizado en un rancho ganadero en Sotuta, Yucatán ( $20^{\circ} 39^{\prime} 14.8^{\prime \prime} \mathrm{N},-89^{\circ} 02^{\prime} 28.8^{\prime \prime} \mathrm{O}, 21 \mathrm{msnm}$ ), en marzo de 2019. Todos los procedimientos de captura y manejo de los animales fueron aprobados por el Comité de Bioética (No. aprobación CB-CCBA I-2018-001) del Campus de Ciencias Biológicas y Agropecuarias de la Universidad Autónoma de Yucatán. La recolección de los roedores contó con el permiso de colecta científica (No. permiso SGPA/DGVS/05995/19) expedido a S. F. Hernández-Betancourt por la Secretaría de Medio Ambiente y Recursos Naturales de México.

Las abreviaciones para las colecciones helmintológicas son las siguientes: CNHE, Colección Nacional de Helmintos, Instituto de Biología, Universidad Nacional Autónoma de México, Ciudad de México, México; HWML, Harold W. Manter Laboratory of Parasitology, University of Nebraska-Lincoln, Nebraska, Estados Unidos de América; USNM, Smithsonian National Museum of Natural History (ahora alberga la U.S. National Parasite Collection), Washington, D.C., Estados Unidos de América; MLP-He, Museo de La PlataColección Helmintológica, La Plata, Argentina; CHE-UAEH, Colección de Helmintos, Universidad Autónoma del Estado de Hidalgo, Hidalgo, México.

\section{Resultados}

La revisión de literatura incluye 111 registros helmintológicos en roedores múridos invasores obtenidos de 18 estudios, incluyendo el reporte realizado en el presente trabajo. Los registros se incluyen en un capítulo de libro, cuatro tesis y 13 artículos científicos realizados desde 1939 hasta mayo de 2020 (Caballero y Caballero 1939; Cerecero 1943a, b Gutiérrez-González 1980; García-Prieto 1986; Hierro-Huerta 1992; Tay Zavala et al. 1999; Pulido-Flores et al. 2005; FalcónOrdaz et al. 2010; Rodríguez-Vivas et al. 2011; García-Prieto et al. 2012; Falcón-Ordaz et al. 2015; Panti-May et al. 2015; Cigarroa-Toledo et al. 2017; Panti-May et al. 2017, 2018; Medina-Pinto et al. 2019). De estos registros, 48 fueron reportados hasta el año 2012 y 63 entre 2013 y 2020. De los 18 estudios, 11 se realizaron hasta el año 2012 (Caballero y Caballero 1939; Cerecero 1943a, b; Gutiérrez-González 1980; García-Prieto 1986; Hierro-Huerta 1992; Tay Zavala et al. 1999; Pulido-Flores et al. 2005; Falcón-Ordaz et al. 2010; Rodríguez-Vivas et al. 2011; García-Prieto et al. 2012) y siete entre los años 2013 y 2020 (Falcón-Ordaz et al. 2015; PantiMay et al. 2015; Cigarroa-Toledo et al. 2017; Panti-May et al. 2017, 2018; Medina-Pinto et al. 2019; presente estudio). Los helmintos fueron registrados en siete de los 32 estados de México (Chiapas, Ciudad de México, Hidalgo, Michoacán, Nuevo León, Tabasco y Yucatán). Los estados con mayor número de taxa de helmintos fueron Tabasco (13 taxa) y Yucatán (12 taxa), mientras que aquellos con mayor número de estudios fueron Yucatán (seis estudios) y Ciudad de México (cinco estudios; Figura 1).

Se identificaron un total de 35 taxa de helmintos en roedores múridos invasores en México. El número de taxa por especie de roedor fue 19 en $M$. musculus, 16 en $R$. norvegicus, 18 en $R$. rattus y dos en Rattus sp. Sin embargo, éstos presentan una asimetría en el número de registros y diversidad entre los estados (Figura 1). Los helmintos reportados incluyeron tremátodos (Diplostomidae y Echinostomatidae), céstodos (Anoplocephalidae, Hymenolepididae y Taeniidae), acantocéfalos (Moniliformidae y Oligacanthorhynchidae) y nematodos (Ascarididae, Gongylonematidae, Heligmonellidae, Heligmosomidae, Heterakidae, Heteroxynematidae, Oxyuridae, Spirocercidae, Strongyloididae, Trichinelloidea y Trichuridae).

Lista parásito-hospedador:

$$
\begin{gathered}
\text { Platyhelminthes Gegenbaur, } 1859 \\
\text { Trematoda Rudolphi, } 1808 \\
\text { Diplostomidae Poirier, } 1886 \\
\text { Fibricola caballeroi Cerecero, } 1943
\end{gathered}
$$

Intestino delgado (adultos). Ciudad de México: Zoológico de Chapultepec, R. norvegicus, CNHE $(802,1372)$, no se reportan datos de prevalencia (Cerecero 1943a).

Echinostomatidae Looss, 1899

Echinostoma revolutum (Frölich, 1802)

Intestino delgado (adulto). Ciudad de México: Zacatenco, Rattus sp., no se reportan datos de material en colección y de prevalencia (Cerecero 1943b).

\section{Euparyphium ochoterenai Cerecero, 1943}

Intestino delgado (adultos). Ciudad de México: Bosque de Chapultepec, $R$. norvegicus, CNHE (800, 1359), no se reportan datos de prevalencia (Cerecero 1943a).

\section{Cestoda Rudolphi, 1808}

Anoplocephalidae Cholodkovsky, 1902

Monoecocestus sigmodontis (Chandler y Suttles, 1922)

Intestino (adultos). Nuevo León: Casas Blancas, el Brasil y la Perla, Apodaca, M. musculus y R. rattus, no se reportan datos de material en colección y de prevalencia (GutiérrezGonzález 1980). 


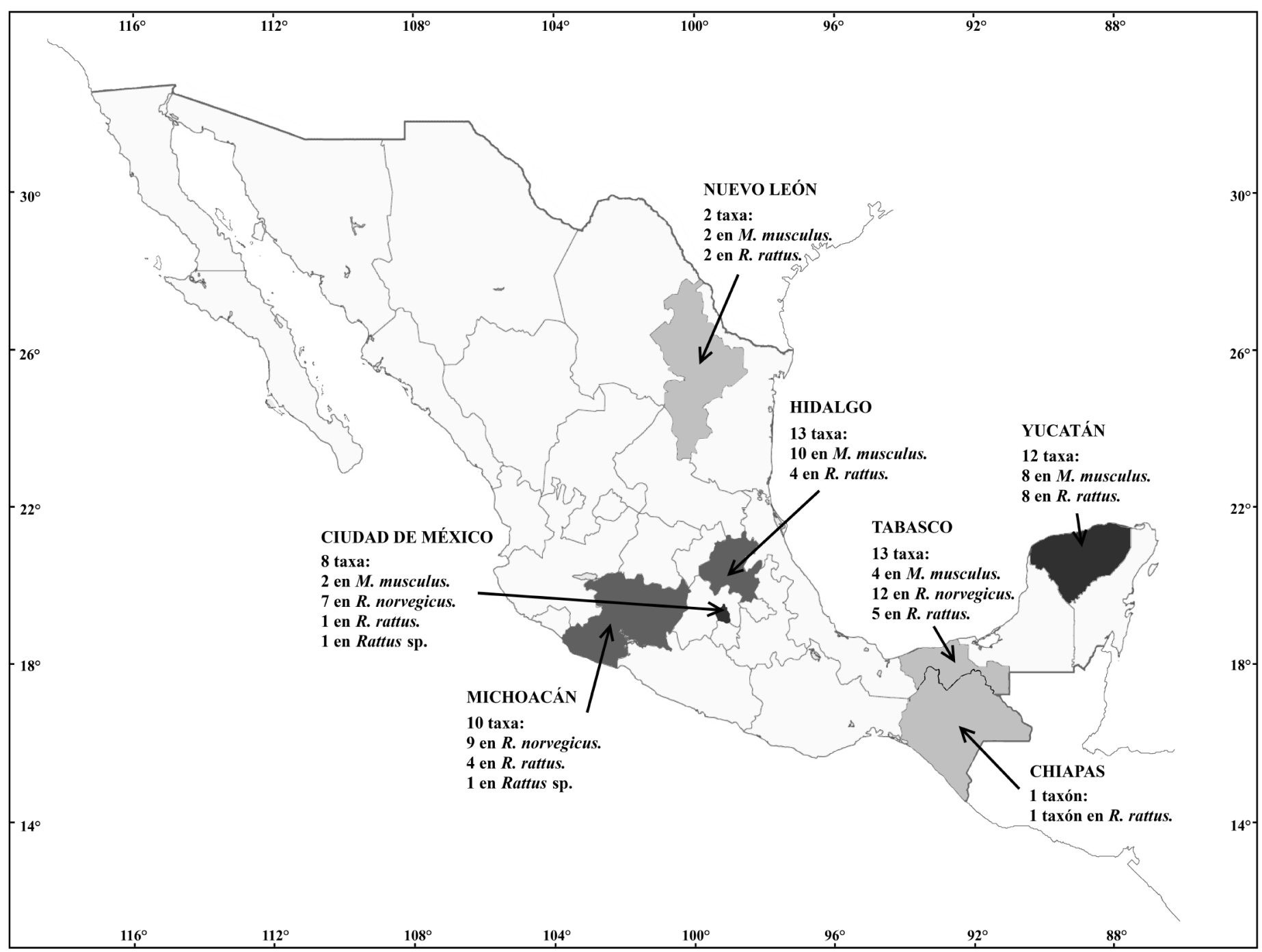

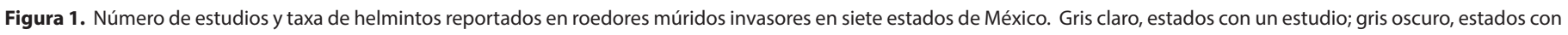
tres estudios; negro, estados con cinco o seis estudios.

Hymenolepididae Ariola, 1899

Hymenolepididae gen. sp. Intestino (adultos).

Chiapas: Pueblo Nuevo, R. rattus, HWML (31364), no se reportan datos de prevalencia (García-Prieto et al. 2012).

Hymenolepis diminuta (Rudolphi, 1819)

Intestino delgado (adultos), heces (huevos). Ciudad de México: no se reporta la localidad, $R$. norvegicus, CNHE (451), no se reportan datos de prevalencia (Caballero y Caballero 1939); Bosque de Chapultepec y rastro de la Ciudad de México, $R$. norvegicus, CNHE $(453,454)$, no se reportan datos de prevalencia (Cerecero 1943b). Michoacán: Ciudad de Morelia, R. norvegicus, CNHE (1316), no se reportan datos de prevalencia (Hierro-Huerta 1992); Ciudad de Morelia, $R$. norvegicus y $R$. rattus, no se reportan datos de material en colección y de prevalencia (Tay Zavala et al. 1999). Tabasco: Ciudad de Villahermosa, R. norvegicus, no se reportan datos de material en colección, 13 \% (3/23; Cigarroa-Toledo et al. 2017). Yucatán: San José Tecoh y Plan de Ayala Sur, Ciudad de Mérida, $R$. rattus, no se reportan datos de material en colección, 14.3 \% (12/84; Panti-May et al. 2017); comunidad de Opichén, Opichén, M. musculus y R. rattus, no se reportan datos de material en colección, $1.9 \%(1 / 52)$ y $23.1 \%$ (3/13; Panti-May et al. 2017); comunidad de Xkalakdzonot, Chankom, R. rattus, MLP-He (7423) y CNHE (10699), $15.2 \%$ (18/118; Panti-May et al. 2018).

\section{Rodentolepis microstoma (Dujardin, 1845)}

Ductos biliares (adultos). Yucatán: Comunidad de Paraíso, Maxcanú, M. musculus, MLP-He (7424) y CNHE (10700), 4.4 \% (7/159; Panti-May et al. 2018).

\section{Rodentolepis nana (von Siebold, 1852)}

Intestino delgado (adultos), heces (huevos). Ciudad de México: No se reporta la localidad, R. norvegicus, CNHE (379, 398-400), no se reportan datos de prevalencia (Caballero y Caballero 1939); Bosque de Chapultepec y rastro de la Ciudad de México, $R$. norvegicus, CNHE (450), no se reportan datos de prevalencia (Cerecero 1943b); no se reporta la localidad, M. musculus, CNHE (381), no se reportan datos de 
prevalencia (García-Prieto 1986). Hidalgo: Meztitlán y San Cristóbal, Reserva de la Biosfera Barranca de Metztitlán, $M$. musculus, CHE-UAEH (F00006), $33.3 \%$ (7/21; Pulido-Flores et al. 2005). Michoacán: Ciudad de Morelia, R. norvegicus, CNHE (1321), no se reportan datos de prevalencia (HierroHuerta 1992); Ciudad de Morelia, $R$. rattus, no se reportan datos de material en colección y de prevalencia (Tay Zavala et al. 1999). Tabasco: Ciudad de Villahermosa, R. norvegicus y $R$. rattus, no se reportan datos de material en colección, $30.4 \%$ (7/23) y $9.1 \%$ (1/11; Cigarroa-Toledo et al. 2017).

Taeniidae Ludwing, 1886

Hydatigera taeniaeformis (Batsch, 1786)

Hígado (larvas). Ciudad de México: No se reporta la localidad, $R$. norvegicus, CNHE (460, 464, 471, 472), no se reportan datos de prevalencia (Caballero y Caballero 1939); no se reporta la localidad, M. musculus, CNHE (685), no se reportan datos de prevalencia (García-Prieto et al. 2012). Hidalgo: San Cristóbal, Reserva de la Biosfera Barranca de Metztitlán, $R$. rattus, CHE-UAEH (P00027), $50 \%$ (2/4; Pulido-Flores et al. 2005). Michoacán: Ciudad de Morelia, R. norvegicus, CNHE (1320), no se reportan datos de prevalencia (Hierro-Huerta 1992). Yucatán: Comunidad de Molas, Mérida, M. musculus y $R$. rattus, no se reportan datos de material en colección, $9 \%$ (29/322) y $3.5 \%$ (2/57; Rodríguez-Vivas et al. 2011); comunidad de Molas, Mérida, M. musculus y R. rattus, MLP-He (6684), $9.7 \%$ (15/154) y 4.3 \% (2/46; Panti-May et al. 2015); San José Tecoh y Plan de Ayala Sur, Ciudad de Mérida, M. musculus y R. rattus, no se reportan datos de material en colección, $5 \%(6 / 121)$ y 14.3 \% (12/84; Panti-May et al. 2017); comunidad de Opichén, Opichén, $M$. musculus y $R$. rattus, no se reportan datos de material en colección, $17.3 \%$ (9/52) y $15.4 \%$ (2/13; PantiMay et al. 2017); comunidad de Xkalakdzonot, Chankom, $M$. musculus y R. rattus, CNHE (10701), $1.3 \%$ (1/74) y $28.8 \%$ (34/118; Panti-May et al. 2018); comunidad de Paraíso, Maxcanú, M. musculus, CNHE (10702), 6.9 \% (11/159; Panti-May et al. 2018); comunidad de Cenotillo, Cenotillo, R. rattus, no se reportan datos de material en colección, 18.5 \% (12/65; Medina-Pinto et al. 2019).

Taenia sp.

Dermis, hígado (larvas). Hidalgo: Huasca, M. musculus, no se reportan datos de material en colección y de prevalencia (Falcón-Ordaz et al. 2015).

Acanthocephala (Rudolphi, 1808)

Moniliformidae Van Cleave, 1924

Moniliformis moniliformis (Bremser, 1811)

Intestino delgado (adultos), heces (huevos). Michoacán: Ciudad de Morelia, $R$. norvegicus, CNHE (617), no se reportan datos de prevalencia (Hierro-Huerta 1992); Ciudad de Morelia, $R$. norvegicus y $R$. rattus, no se reportan datos de material en colección y de prevalencia (Tay Zavala et al.
1999). Tabasco: Ciudad de Villahermosa, $R$. norvegicus, no se reportan dados de material en colección, $8.7 \%(2 / 23$; Cigarroa-Toledo et al. 2017).

\section{Oligacanthorhynchidae Southwell y MacFie, 1925}

Oligacanthorhynchidae gen. sp.

Intestino delgado (adultos). Yucatán: Comunidad de Xkalakdzonot, Chankom, R. rattus, MLP-He (7426), $0.8 \%$ (1/118; Panti-May et al. 2018).

\section{Nematoda Rudolphi, 1808 \\ Ascarididae Baird, 1853 \\ Toxocara sp.}

Heces (huevos). Tabasco: Ciudad de Villahermosa, $R$. norvegicus, no se reportan datos de material en colección, $4.3 \%$ (1/23; Cigarroa-Toledo et al. 2017).

Gongylonematidae (Hall, 1916)

Gongylonema neoplasticum (Fibiger y Ditlevsen, 1914).

Estómago (adultos). Michoacán: Ciudad de Morelia, $R$. norvegicus, CNHE (2392), no se reportan datos de prevalencia (Hierro-Huerta 1992). Yucatán: Comunidad de Xkalakdzonot, Chankom, M. musculus y R. rattus, MLP-He (7438, 7441) y CNHE (10710), 6.7 \% (5/75) y $11.9 \%$ (14/118; PantiMay et al. 2018).

\section{Gongylonema sp.}

Estómago (adultos). Hidalgo: Meztitlán, Reserva de la Biosfera Barranca de Metztitlán, M. musculus, CHE-UAEH (F00002), $33.3 \%$ (1/3; Pulido-Flores et al. 2005).

Heligmonellidae (Skrjabin y Schikhobalova, 1952)

Hassalstrongylus aduncus (Chandler, 1932).

Intestino delgado (adultos). Yucatán: Comunidad de Xkalakdzonot, $R$. rattus, MLP-He (7431) y CNHE (10713), $1.7 \%$ (2/118; Panti-May et al. 2018).

Hassalstrongylus musculi (Dickmans, 1935).

Intestino delgado (adultos). Yucatán: Comunidad de Paraíso, Maxcanú, M. musculus, MLP-He (7432) y CNHE (10711), 17 \% (27/159; Panti-May et al. 2018); Rancho Kuncheil, Sotuta, M. musculus, CNHE (11050), 100 \% (5/5; presente estudio).

Nippostrongylus brasiliensis (Travassos, 1914).

Intestino delgado (adultos), heces (huevos). Hidalgo: San Cristóbal, Reserva de la Biosfera Barranca de Metztitlán, $R$. rattus, CHE-UARH (F00007) y CNHE (5320), 100 \% (4/4; Pulido-Flores et al. 2005). Michoacán: Ciudad de Morelia, $R$. norvegicus, CNHE (2391), no se reportan datos de prevalencia (Hierro-Huerta 1992). Tabasco: Ciudad de Villahermosa, 
$M$. musculus, $R$. norvegicus y $R$. rattus, no se reportan datos de material en colección, $6.2 \%$ (1/16), $34.8 \%$ (8/23) y $18.2 \%$ (2/11; Cigarroa-Toledo et al. 2017). Yucatán: Comunidad de Molas, Mérida, M. musculus y R. rattus, MLP-He (6687), 81.2 \% (125/154) y 43.5 \% (20/46; Panti-May et al. 2015); San José Tecoh y Plan de Ayala Sur, Ciudad de Mérida, M. musculus y $R$. rattus, no se reportan datos de material en colección, 28.1 \% (34/121) y 4.8 \% (4/84; Panti-May et al. 2017); comunidad de Opichén, Opichén, $M$. musculus y $R$. rattus, no se reportan datos de material en colección, $21.1 \%(11 / 52)$ y 7.7 \% (1/13; Panti-May et al. 2017); comunidad de Paraíso, Maxcanú, $R$. rattus, MLP-He (7433) y CNHE (10712), $14.3 \%$ (1/7; Panti-May et al. 2018).

Heligmosomidae (Travassos, 1914)

Heligmosomoides polygyrus (Dujardin, 1845).

Heces (huevos). Tabasco: Ciudad de Villahermosa, $R$. norvegicus y $R$. rattus, no se reportan datos de material en colección, $56.5 \%$ (13/23) y $36.4 \%$ (4/11; Cigarroa-Toledo et al. 2017).

\section{Longistriata sp.}

Intestino (adultos). Nuevo León: Casas Blancas, el Brasil y la Perla, Apodaca, M. musculus y $R$. rattus, no se reportan datos de material en colección y de prevalencia (GutiérrezGonzález 1980).

Heterakidae Ralliet y Henry, 1914

Heterakis spumosa Schneider, 1866.

Intestino grueso (adultos), estómago (adultos), heces (huevos). Ciudad de México: no se reporta la localidad, $R$. norvegicus, CNHE (2003), no se reportan datos de prevalencia (Caballero y Caballero 1939); Bosque de Chapultepec, $R$. norvegicus, CNHE (2001, 2002), no se reportan datos de prevalencia (García-Prieto et al. 2012). Michoacán: Ciudad de Morelia, $R$. norvegicus, CNHE (2393), no se reportan datos de prevalencia (Hierro-Huerta 1992). Tabasco: Ciudad de Villahermosa, $R$. norvegicus, no se reportan datos de material en colección, 13 \% (3/23; Cigarroa-Toledo et al. 2017).

Heteroxynematidae Skrjabin y Shikhobalova, 1948

Aspiculuris huascaensis Falcón-Ordáz, Monks y PulidoFlores, 2010.

Ciego (adultos). Hidalgo: Cerro del Tezontle y San Juan Hueyapan, Huasca de Ocampo, M. musculus, CNHE (6395-7) y HWML (64565), 13.8 \% (4/29; Falcón-Ordaz et al. 2010).

Aspiculuris cf. lahorica Akhtar, 1955.

Ciego (adultos). Hidalgo: Meztitlán, Reserva de la Biosfera Barranca de Metztitlán, M. musculus, CHE-UAEH (F00001), 33.3 \% (1/3; Pulido-Flores et al. 2005).
Aspiculuris sp.

Heces (huevos). Yucatán: San José Tecoh y Plan de Ayala Sur, Ciudad de Mérida, $R$. rattus, no se reportan datos de material en colección, 8.3 \% (7/84; Panti-May et al. 2017).

Oxyuridae Cobbold, 1864

Syphacia muris (Yamaguti, 1935).

Intestino grueso (adultos), heces (huevos). Hidalgo: San Cristóbal, Reserva de la Biosfera Barranca de Metztitlán, $R$. rattus, CHE-UAEH (F00008) y CNHE (5319), 50 \% (2/4; PulidoFlores et al. 2005). Tabasco: Ciudad de Villahermosa, R. rattus, no se reportan datos de material en colección, $27.3 \%$ (3/11; Cigarroa-Toledo et al. 2017). Yucatán: Comunidad de Molas, Mérida, R. rattus, MLP-He (6686), 17.4 \% (8/46; PantiMay et al. 2015); San José Tecoh y Plan de Ayala Sur, Ciudad de Mérida, $R$. rattus, no se reportan datos de material en colección, 17.9 \% (15/84; Panti-May et al. 2017); comunidad de Opichén, Opichén, $R$. rattus, no se reportan datos de material en colección, $15.4 \%$ (2/13; Panti-May et al. 2017); comunidad de Xkalakdzonot, Chankom, R. rattus, MLP-He (7435) y CNHE (10706), 65.3 \% (77/118; Panti-May et al. 2018).

\section{Syphacia obvelata (Rudoplhi, 1802).}

Intestino grueso (adultos), heces (huevos). Hidalgo: San Cristóbal y Metztitlán, Reserva de la Biosfera Barranca de Metztitlán, M. musculus, CHE-UAEH (F00003) y CNHE (5318), $28.6 \%$ (6/21; Pulido-Flores et al. 2005). Tabasco: Ciudad de Villahermosa, $M$. musculus y $R$. norvegicus, no se reportan datos de material en colección, $6.2 \%(1 / 16)$ y $4.3 \%(1 / 23$; Cigarroa-Toledo et al. 2017). Yucatán: Comunidad de Opichén, Opichén, M. musculus, no se reportan datos de material en colección, 9.6 \% (5/52; Panti-May et al. 2017); comunidad de Paraíso, Maxcanú, M. musculus, MLP-He (7434) y CNHE (10708), 3.8 \% (6/159; Panti-May et al. 2018).

\section{Syphacia sp. Ciego (adultos).}

Hidalgo: Cerro del Tezontle y San Juan Hueyapan, Huasca de Ocampo, M. musculus y $R$. rattus, no se reportan datos de material en colección y de prevalencia (FalcónOrdaz et al. 2015).

\section{Spirocercidae (Chitwood y Wehr, 1933) \\ Mastophorus muris (Gmelin, 1790).}

Estómago (adultos), heces (huevos). Michoacán: Uruapan, Rattus sp., USNM (1345984), no se reportan datos de prevalencia (García-Prieto et al. 2012). Tabasco: Ciudad de Villahermosa, $R$. norvegicus, no se reportan datos de material en colección, 17.4 \% (4/23; Cigarroa-Toledo et al. 2017).

Strongyloididae Chitwood y McIntosh, 1934

Strongyloides ratti Sandground, 1925.

Heces (huevos). Tabasco: Ciudad de Villahermosa, $M$. 
musculus, $R$. norvegicus y $R$. rattus, no se reportan datos de material en colección, $12.5 \%$ (2/16), $65.2 \%$ (15/23) y $45.5 \%$ (5/11; Cigarroa-Toledo et al. 2017).

Trichosomoides crassicauda (Bellingham, 1840).

Heces (huevos). Tabasco: Ciudad de Villahermosa, $R$. norvegicus, no se reportan datos de material en colección, $8.7 \%$ (2/23; Cigarroa-Toledo et al. 2017).

Trichuridae (Ransom, 1911)

Capillaria sp.

Vejiga urinaria (adultos). Michoacán: Ciudad de Morelia, R. norvegicus, CNHE (2394), no se reportan datos de prevalencia (Hierro-Huerta 1992).

\section{Trichinella spiralis (Owen, 1835).}

Músculo (larvas). Michoacán: Ciudad de Morelia, R. norvegicus y $R$. rattus, no se reportan datos de material en colección y de prevalencia (Tay Zavala et al. 1999).

\section{Trichinella sp. Músculo (larvas).}

Hidalgo: San Cristóbal, Reserva de la Biosfera Barranca de Metztitlán, M. musculus, CHE-UAEH (F00004), $5.5 \%$ (1/18; Pulido-Flores et al. 2005).

\section{Trichuris muris (Schrank, 1788).}

Intestino, ciego (adultos), heces (huevos). Ciudad de México: Bosque de Chapultepec, $R$. norvegicus, CNHE (2399), no se reportan datos de prevalencia (Cerecero 1943b); no se reporta la localidad, $R$. rattus, CNHE (2247), no se reportan datos de prevalencia (García-Prieto et al. 2012). Hidalgo: San Cristóbal y Meztitlán, Reserva de la Biosfera Barranca de Metztitlán, M. musculus, CNHE (5316) y CHE-UAEH (F00005), $9.5 \%$ (2/21; Pulido-Flores et al. 2005). Tabasco: Ciudad de Villahermosa, M. musculus y R. norvegicus, no se reportan datos de material en colección, $6.3 \%$ (1/16) y 13 \% (3/23; Cigarroa-Toledo et al. 2017). Yucatán: Comunidad de Molas, Mérida, M. musculus, MLP-He (6685), 11.7 \% (18/154; Panti-May et al. 2015); San José Tecoh y Plan de Ayala Sur, Ciudad de Mérida, M. musculus, no se reportan datos de material en colección, $13.2 \%$ (16/121; Panti-May et al. 2017); comunidad de Opichén, Opichén, M. musculus, no se reportan datos de material en colección, 32.7 \% (17/52; Panti-May et al. 2017); comunidad de Paraíso, Maxcanú, $M$. musculus, MLP-He (7427) y CNHE (10703), 15.1 \% (24/159; Panti-May et al. 2018).

\section{Trichuris sp.}

Ciego (adultos). Hidalgo: Cerro del Tezontle y San Juan Hueyapan, Huasca de Ocampo, M. musculus, no se reportan datos de material en colección y de prevalencia (FalcónOrdaz et al. 2015).

\section{Discusión}

Los roedores múridos invasores están presentes a lo largo del territorio nacional en áreas rurales, urbanas e incluso en áreas naturales como islas. Los estudios helmintológicos en estos roedores han sido escasos en comparación, por ejemplo, con aquellos realizados con roedores cricétidos o heterómidos (ver listados helmintológicos de García-Prieto et al. 2012; Preissar y Falcón-Ordaz 2019). La presente revisión actualiza el número de helmintos registrados en roedores múridos a 35 taxa identificados en siete estados de México, incluyendo tremátodos, céstodos, acantocéfalos y nematodos. De éstos, 12 fueron reportados posteriormente a la publicación del listado helmintológico de García-Prieto et al. (2012). Para M. musculus, R. norvegicus y $R$. rattus, el número de taxa de helmintos se actualizó a 19, 16 y 18 , respectivamente. Anteriormente, García-Prieto et al. (2012) reportaron 10 taxa para Mus y 12 para Rattus. A pesar de esto, es probable que el número de especies de helmintos que estos roedores albergan esté subestimado debido al escaso número de estudios realizados en estos hospedadores, el número de especímenes y especies de hospedadores examinados, así como el número de taxa que no han sido identificados a nivel especie.

La mayoría de las especies de helmintos registradas en los roedores múridos en México son específicas de estos roedores, y por lo general ocasionan infecciones leves no patogénicas y no causan signos clínicos en los animales (Taffs 1976; Baker 2007). Sin embargo, algunas especies de helmintos pueden ser introducidas con sus hospedadores invasores y afectar negativamente la salud de hospedadores no naturales como los roedores silvestres. Hydatigera taeniaeformis, un parásito del gato doméstico (hospedador definitivo) y roedores múridos (hospedadores intermediarios), ha sido mencionado como el causante de muertes masivas en Ondrata zibethicus en Republica Checa (Dvorakova y Prokopic 1984). En México, esta especie ha sido reportada en los roedores silvestres Sigmodon hispidus en Nuevo León (Gutiérrez-González 1980), S. toltecus en Yucatán (Panti-May et al. 2018) y en Sciurus yucatanensis en Yucatán (Panti-May et al. 2019) usualmente con intensidades de infección mayores (intensidad media 19.7, rango 1-50, Panti-May et al. 2018, 2019) a las reportadas en roedores múridos (intensidad media 1.1-1.5, rango 1-4; RodríguezVivas et al. 2011; Panti-May et al. 2015; 2018; Medina-Pinto et al. 2019), debido probablemente a una respuesta inmune más fuerte de los roedores múridos hacia este cestodo (Rodríguez-Vivas et al. 2011). También, se ha reportado la introducción de T. muris con su hospedador $R$. rattus en localidades donde viven poblaciones de Peromyscus maniculatus, en las Islas del Mar de Cortés (Smith y Carpenter 2006), aunque sin estudios del impacto en la salud de los roedores silvestres.

El listado helmintológico de roedores múridos invasores en México mostró una mayor diversidad de nematodos en comparación con los tremátodos, céstodos o acantocéfalos. Esto puede estar relacionado con el ciclo de vida 
directo de la mayoría de las especies de nematodos registradas, con pocas excepciones como G. neoplasticum y $T$. spiralis que tiene un ciclo de vida indirecto. En parásitos con ciclo de vida directo, la infección y distribución de los parásitos está determinada por factores micro-ambientales favorables (e. g., temperatura, humedad) para los estadios infectantes, así como la distribución y abundancia de los hospedadores (Arneberg 2001; Wilson et al. 2002). La mayor diversidad de nematodos puede estar relacionada con las características estables de los asentamientos humanos que favorecen la transmisión de los nematodos en las poblaciones roedores múridos que generalmente son abundantes. En contraste, las especies de céstodos (con excepción de $R$. nana) y acantocéfalos reportadas requieren de un hospedador intermediario (e. g., escarabajos, cucarachas, pulgas, e incluso roedores) para completar su ciclo de vida, y los tremátodos requieren de dos hospedadores intermediarios (e. g., moluscos y anfibios). Considerando que factores como la abundancia de los hospedadores intermediarios y hábitos alimenticios de los hospedadores definitivos afectan la transmisión de los helmintos con ciclo de vida indirecto (Krasnov et al. 2006), es posible que en las áreas muestreadas estos factores presenten variaciones marcadas que promuevan una baja transmisión para algunas especies de tremátodos, céstodos o acantocéfalos. Sin embargo, es necesario estudiar los múltiples factores que determinan los patrones de infección de los helmintos que parasitan roedores múridos en México.

Aunque el listado de helmintos que parasitan roedores múridos invasores en México incluye más de 30 taxa, la mayoría de éstos presentan pocos registros. Considerando el número de especies de hospedadores infectadas y la distribución en el país, siete especies de helmintos podrían considerase como las más frecuentes, incluyendo céstodos ( $H$. taeniaeformis, $H$. diminuta y $R$. nana) y nematodos ( $N$. brasiliensis, S. obvelata, S. muris y T. muris). Estas especies han sido reportadas también como frecuentes en estos roedores en distintas regiones del mundo (Oldham 1931; McKenna 1997; Gliga et al. 2020).

Los registros helmintológicos mostraron una asimetría en el número de registros y diversidad de helmintos entre las tres especies de roedores de los siete estados (ver Figura 1). Por ejemplo, en la única especie, $R$. rattus, con registros en los siete estados, el número de especies de helmintos varió de uno a siete considerando los diferentes estudios, y de uno a ocho considerando los estados. Estas variaciones pueden deberse a múltiples factores como el número de estudios y hospedadores examinados, el periodo de colecta, así como la abundancia de los hospedadores (intermediarios y definitivos) y características micro-ambientales presentes en las áreas de colecta (selvas, ranchos, comunidades rurales, ciudades).

Uno de los motivos que ha favorecido el incremento de los estudios parasitológicos en los roedores múridos invasores en los últimos años, es la investigación sobre patógenos zoonóticos (e. g., helmintos) presentes en sus poblaciones sinantrópicas. En México, los estudios helmintológicos han reportado en los roedores algunas especies de tremátodos (E. revolutum), céstodos ( $H$. diminuta, $R$. nana, $R$. microstoma, $H$. taeniaeformis), acantocéfalos ( $M$. moniliformis) y nematodos (T. spiralis) que pueden transmitirse directa o indirectamente a los humanos (Macnish et al. 2003; Larrieu et al. 2004; Salehabadi et al. 2008; Sohn et al. 2011; Panti-May et al. 2020a; $\underline{b}$ ). Entre estos resaltan los hymenolepididos R. nana e $H$. diminuta que causan la hymenolepiasis humana, principalmente en niños de áreas con carencias socioeconómicas. El primero es el céstodo más común en humanos con una prevalencia mundial estimada de $4 \%$ (Bogitsh et al. 2012), mientras que el segundo cestodo tiene más de 500 registros en humanos entre 2000-2018 (Panti-May et al. 2020b). La relevancia de estos hospedadores en asentamientos humanos como ciudades o comunidades rurales se debe a sus poblaciones abundantes y el contacto frecuente con los habitantes, lo que puede favorecer la transmisión de patógenos zoonóticos (Panti-May et al. 2012).

Basados en el listado de helmintos registrados en $M$. musculus, $R$. norvegicus y $R$. rattus, es evidente que la diversidad de especies de helmintos en roedores múridos invasores en México aún está subestimada. En menos del $25 \%$ de los estados de México se han estudiado los helmintos de estos roedores, y además, el número de hospedadores y localidades muestreados en cada estudio han sido heterogéneos. Es necesario realizar más estudios para incrementar el conocimiento de los helmintos que albergan los roedores múridos invasores, así como su distribución en el país, en especial considerando la presencia de especies zoonóticas.

\section{Agradecimientos}

El muestreo en Sotuta fue financiado por el CONACYT a través del proyecto "Análisis y evaluación de los probables vectores y reservorios del virus del Ebola en México", CONACYT-251053, proyecto apoyado por el Fondo Sectorial de Investigación para la Educación (SFH-B). A tres revisores anónimos del manuscrito original por sus valiosos comentarios y sugerencias que enriquecieron el trabajo.

\section{Literatura citada}

Arneberg, P. 2001. An ecological law and its macroecological consequences as revealed by studies of relationships between host densities and parasite prevalence. Ecography 24:352-358.

BakeR, D. G. 2007. Parasites of rats and mice. Pp. 303-397, in Flynn's parasites of laboratory animals (Baker, D. G., ed.). Blackwell Publishing. lowa, U.S.A.

Bogitsh, B. J., C. E. Carter, y T. N. Oeltmann. 2012. Human parasitology. Academic Press. New York, U.S.A.

Burgin, C. J., J. P. Colella, P. L. Kahn, y N. S. Upham. 2018. How many species of mammals are there? Journal of Mammalogy 99:1-14.

Caballero y Caballero, E. 1939. Algunos endoparásitos de Rattus rattus norvegicus y de Rattus norvegicus albinus, del Laboratorio de Investigaciones Médicas del Hospital General de la Ciudad de México. Anales del Instituto de Biología 10:285-291. 
Cerecero, M. C. 1943a. Algunos helmintos de las ratas domésticas y silvestres de México con descripción de dos nuevas especies. Lic. Tesis, Facultad de Ciencias, Universidad Nacional Autónoma de México, México.

Cerecero, M. C. 1943b. Algunos trematodos de las ratas domésticas de la Ciudad de México. Anales del Instituto de Biología 14:507-526.

Chitwood, B. G. 1938. Some nematodes from the caves of Yucatan. Publications of Carnegie Institute of Washington 491:51-66.

Cigarroa-Toledo, N., Y. Santos-Martinez, C. V. Zaragoza-Vera, M. M. Garcia-Rodriguez, C. M. BaAK-BaAk, C. Machain-Williams, J. E. Garcia-Rejon, J. A. Panti-May, y O. M. Torres-Chable. 2017. Occurrence of gastrointestinal helminths in commensal rodents from Tabasco, Mexico. Helminthologia 54:170-173.

Dvotakova, L., y J. Prokopic. 1984. Hydatigera taeniaeformis (Batsch, 1786) as the cause of mass deaths of muskrats. Folia Parasitologica 31:127-131.

Falcón-Ordaz, J., S. Monks, G. Pulido-Flores, L. García-Prieto, y G. Lira-Guerrero. 2015. Riqueza de helmintos parásitos de vertebrados silvestres del estado de Hidalgo, México. Pp. 2037, in Estudios de Biodiversidad: Volumen I (Pulido-Flores, G. , S. Monks y M. López-Herrera, eds.). Zea Books. Lincoln, U.S.A. Falcón-Ordaz, J., G. Pulido-Flores, y S. Monks. 2010. New species of Aspiculuris (Nematoda: Heteroxynematidae), parasite of Mus musculus (Rodentia: Muridae), from Hidalgo, Mexico. Revista Mexicana de Biodiversidad 81:669-676.

García-Prieto, L. 1986. Estudio taxonómico de algunos céstodos de vertebrados de México. Lic. Tesis, Facultad de Ciencias, Universidad Nacional Autónoma de México, México.

García-Prieto, L., J. Falcón-Ordaz, y C. Guzmán-Cornejo. 2012. Helminth parasites of wild Mexican mammals: list of species, hosts and geographical distribution. Zootaxa 92:1-92.

Gliga, D. S., B. Pisanu, C. Walzer, y A. Desvars-Larrive. 2020. Helminths of urban rats in developed countries: a systematic review to identify research gaps. Parasitology Research 119:2383-2397.

GutiérRez-GonzÁLez, J. 1980. Algunos helmintos parásitos en ratas silvestres de Apodaca, Nuevo León, México. Lic. Tesis, Facultad de Ciencias Biológicas, Universidad Autónoma de Nuevo León, México.

Hierro-Huerta, P. 1992. Helmintofauna de la rata de alcantarilla Rattus norvegicus Erxleben 1777, de la Ciudad de Morelia, Michoacán, México. Lic. Tesis, Escuela de Biología, Universidad Michoacana de San Nicolás de Hidalgo, México.

Krasnov, B. R., R. Poulin, y S. Morand. 2006. Patterns of macroparasite diversity in small mammals. Pp. 197-231, in Micromammals and macroparasites: From evolutionary ecology to management (Morand, S., B. R. Krasnov y R. Poulin, eds.). Springer. Tokyo, Japan.

Larrieu, E., V. Molina, S. Albarkacín, S. Mancini, R. Bigatti, L. Ledesma, C. Chiosso, S. Krivokapich, E. Herero, y E. Guarnera. 2004. Porcine and rodent infection with Trichinella, in the Sierra Grande area of Rio Negro province, Argentina. Annals of Tropical Medicine and Parasitology 98:725-731.

Leitschum, C. M., D. Kanavy, G. A. Backus, R. X. Valdez, M., Serr, E. A. Pitts, D. Threadgill, y J. Godwin. 2018. Developing gene drive technologies to eradicate invasive rodents from islands. Journal of Responsible Innovation 5:S121-S138.
Macnish, M. G., U. M. Ryan, J. M. Behnke, y R. C. A. Thompson. 2003. Detection of the rodent tapeworm Rodentolepis $(=\mathrm{Hy}$ menolepis) microstoma in humans. A new zoonosis? International Journal for Parasitology 33:1079-1085.

MckennA, P. B. 1997. Checklist of helminth parasites of terrestrial mammals in New Zealand. New Zealand Journal of Zoology 24:277-290.

Medina-Pinto, R. A., M. A. Torres-Castro, R. A. Medina-Pinto, M. E. Bolio-González, y R. I. Rodríguez-Vivas. 2019. Natural Cysticercus fasciolaris infection in rodents from a rural area in Yucatan, Mexico. Veterinaria México 6:1-10.

Meerburg, B. G., G. R. Singleton, y A. KiJlstra. 2009. Rodentborne diseases and their risks for public health. Critical Reviews in Microbiology 35:221-270.

Ochoterena, I., y E. Caballero y Caballero. 1932. Filaria parásita de las ratas de campo Micropleura sigmodoni spec., nov. Anales del Instituto de Biología 3:123-125.

Oldham, J. N. 1931. The helminth parasites of common rats. Journal of Helminthology 9:49-90.

Panti-May, J. A., M. C. Digiani, E. E. Palomo-Arjona, Y. M. Gurubel-Gonzalez, G. T. Navone, C. Machain-Williams, S. F. Hernández-Betancourt, y M. R. Robles. 2018. A checklist of the helminth parasites of sympatric rodents from two Mayan villages in Yucatán, México. Zootaxa 4403:495-512.

Panti-May, J. A., E. E. Palomo-Arjona, Y. M. Gurubel-Gonzalez, M. A. Torres-Castro, M. V. Vidal-Martínez, C. Machain-Williams, S. F. Hernández-Betancourt, y M. R. Robles. 2017. New host, geographical records, and factors affecting the prevalence of helminths infection from synanthropic rodents in Yucatán, Mexico. Helminthologia 54:231-239.

Panti-May, J. A., A. Servían, W. Ferreri, M. L. Zonta, D. I. Hernández-Mena, S. F. Hernández-Betancourt, M. R. Robles, y C. Machain-Williams. 2020A. Morphological and molecular identification of hymenolepidid cestodes in children and synanthropic rodents from rural Mexico. Parasitology International 75:102042.

Panti-May, J. A., S. F. Hernández-Betancourt, y L. García-Prieto. 2019. Hydatigera taeniaeformis (Cestoda: Taeniidae) in the Yucatán squirrel Sciurus yucatanensis (Rodentia: Sciuridae), Mexico. Therya 10:179-182.

Panti-May, J. A., S. F. Hernández-Betancourt, R. I. RodríguezVivas, y M. R. Robles. 2015. Infection levels of intestinal helminths in two commensal rodent species from rural households in Yucatan, Mexico. Journal of Helminthology 89:42-48.

Panti-May, J. A., S. Hernández-Betancourt, H. Ruíz-Piña, y S. Medina-Peralta. 2012. Abundance and population parameters of commensal rodents present in rural households in Yucatan, Mexico. International Biodeterioration and Biodegradation 66:77-81.

Panti-May, J. A., R. I. Rodríguez-Vivas, L. García-Prieto, A. Servián, y F. CostA. 2020B. Worldwide overview of human infections with Hymenolepis diminuta. Parasitology Research 119:1997-2004.

Preisser, W. C., y J. Falcón-Ordaz. 2019. A checklist of the parasitic helminths of cricetid and heteromyid rodents in Mexico. Therya 10:329-341.

Pulido-Flores, G., S. Moreno-Flores, y S. Monks. 2005. Helminths of rodents (Rodentia: Muridae) from Metztitlán, San Cristobal, and Rancho Santa Elena, Hidalgo, Mexico. Comparative Parasitology 72:186-192. 
Rodríguez-Vivas, R. I., J. A. Panti-May, J. Parada-López, S. F. Hernández-Betancourt, y H. Ruiz-Piña. 2011. The occurrence of the larval cestode Cysticercus fasciolaris in rodent populations from the Cuxtal ecological reserve, Yucatan, Mexico. Journal of Helminthology 85:458-461.

Salehabadi, A., G. Mowlavi, y S. M. Sadjuadi. 2008. Human infection with Moniliformis moniliformis (Bremser 1811) (Travassos 1915) in Iran: another case report after three decades. Vector-Borne and Zoonotic Diseases 8:101-104.

Singleton, G. R., S. Belmain, P. R. Brown, K. Aplin, y N. M. Htwe. 2010. Impacts of rodent outbreaks on food security in Asia. Wildlife Research 37:355-359.

Smith, K. F., K. F. Smith, S. M. Carpenter, y S. M. Carpenter. 2006. Potential spread of introduced black rat (Rattus rattus) parasites to endemic deer mice (Peromyscus maniculatus) on the California Channel Islands. Diversity and Distributions:742-748.

Sohn, W. M., J. Y. Chal, T. S. Yong, K S. Eom, C. H. Yoon, M. Sinuon, D. Socheat, y S. H. Lee. 2011. Echinostoma revolutum infection in children, Pursat Province, Cambodia. Emerging Infectious Diseases 17:117-119.

Stenseth, N. C., H. Leirs, A. Skonhoft, S. A. Davis, R. P. Pech, H. P. Andreassen, G. R. Singleton, M. Lima, R. M. Machangu, R. H. Makundi, Z. Zhang, P. B. Brown, D. Shi, y X. Wan. 2003. Mice and rats: the dynamics and bio- economics of agricultural rodents pests. Frontiers in Ecology and the Environment 1:367-375.

Taffs, L. F. 1976. Pinworm infections in laboratory rodents: a review. Laboratory Animals 10:1-13.

Tay Zavala, J., T. Alvarez, M. Gutiérrez Quiroz, J. Sánchez Vega, y A. Fenández Presas. 1999. Zoonosis por helmintos en mamíferos de Morelia, Michoacán, República Mexicana. Revista de la Facultad de Medicina 42:64-65.

Wilson, K., O.N. Buornstad, A.P. Dobson, S. Merler, G. Poglayen, S.E. Randolph, y A. SKorping. 2002. Heterogeneities in macroparasite infections: patterns and processes. Pp. 6-44, in The Ecology of Wildlife Diseases (Hudson, P. J., A. Rizzoli, B. T. Grenfell, H. Heesterbeek y A. P. Dobson, eds.). Oxford University Press. New York, U.S.A.

\section{Associated editor: Jesús Fernández}

Submitted: July 17, 2020; Reviewed: August 11, 2020;

Accepted: October 30, 2020; Published on line: November 20, 2020. 
94 TTHERYA Vol. 12 (1):85-93 